\title{
Simple and Fast Derandomization from Very Hard Functions: Eliminating Randomness at Almost No Cost
}

\author{
Lijie Chen \\ lijieche@mit.edu \\ Massachusetts Institute of Technology \\ Cambridge, MA, USA
}

\author{
Roei Tell \\ roeitell@gmail.com \\ Massachusetts Institute of Technology \\ Cambridge, MA, USA
}

\begin{abstract}
Extending the classical "hardness-to-randomness" line-of-works, Doron, Moshkovitz, Oh, and Zuckerman (FOCS 2020) recently proved that derandomization with near-quadratic time overhead is possible, under the assumption that there exists a function in $\mathcal{D T} \mathcal{I} \mathcal{M E}\left[2^{n}\right]$ that cannot be computed by randomized SVN circuits of size $2^{(1-\epsilon) \cdot n}$ for a small $\epsilon$.

In this work we extend their inquiry and answer several open questions that arose from their work. For a time function $T(n)$, consider the following assumption: Non-uniformly secure one-way functions exist, and for $\delta=\delta(\epsilon)$ and $k=k_{T}(\epsilon)$ there exists a problem in $\mathcal{D T} \mathcal{I} \mathcal{M E}\left[2^{k \cdot n}\right]$ that is hard for algorithms that run in time $2^{(k-\delta) \cdot n}$ and use $2^{(1-\delta) \cdot n}$ bits of advice. Under this assumption, we show that:
\end{abstract}

(1) (Worst-case derandomization.) Probabilistic algorithms that run in time $T(n)$ can be deterministically simulated in time $n \cdot T(n)^{1+\epsilon}$.

(2) (Average-case derandomization.) For polynomial time functions $T(n)=\operatorname{poly}(n)$, we can improve the derandomization time to $n^{\epsilon} \cdot T(n)$ if we allow the derandomization to succeed only on average, rather than in the worst-case.

(3) (Conditional optimality.) For worst-case derandomization, the multiplicative time overhead of $n$ is essentially optimal, conditioned on a counting version of the non-deterministic strong exponential-time hypothesis (i.e., on \#NSETH).

Lastly, we present an alternative proof for the result of Doron, Moshkovitz, Oh, and Zuckerman that is simpler and more versatile. In fact, we show how to simplify the analysis not only of their construction, but of any construction that "extracts randomness from a pseudoentropic string".

\section{CCS CONCEPTS}

- Theory of computation $\rightarrow$ Circuit complexity; Complexity classes; Pseudorandomness and derandomization.

\section{KEYWORDS}

circuit lower bounds, derandomization, hardness versus randomness

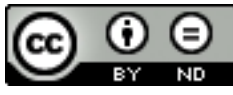

This work is licensed under a Creative Commons Attribution-NoDerivs International 4.0 License.

STOC '21, fune 21-25, 2021, Virtual, Italy

(c) 2021 Copyright held by the owner/author(s)

ACM ISBN 978-1-4503-8053-9/21/06.

https://doi.org/10.1145/3406325.3451059
ACM Reference Format:

Lijie Chen and Roei Tell. 2021. Simple and Fast Derandomization from Very Hard Functions: Eliminating Randomness at Almost No Cost. In Proceedings of the 53rd Annual ACM SIGACT Symposium on Theory of Computing (STOC '21), fune 21-25, 2021, Virtual, Italy. ACM, New York, NY, USA, 9 pages. https://doi.org/10.1145/3406325.3451059

\section{INTRODUCTION}

Can we replace all randomized algorithms for decision problems by deterministic algorithms with roughly similar runtime? The long line of works typically referred to as "hardness-to-randomness", which was initiated by $[5,19,30]$, gives one way of answering the foregoing question: These works show that certain lower bounds for non-uniform circuits imply derandomization with bounded runtime overhead.

The fastest conditional derandomization in the classical line-ofworks was proved by Impagliazzo and Wigderson [15], who showed that if $\mathcal{E} \not \subset S I Z E\left[2^{.01 \cdot n}\right]$, then $\operatorname{pr} \mathcal{B P} \mathcal{P}=p r \mathcal{P}$. By a padding argument, this conclusion implies that randomized time- $T$ algorithms can be simulated in deterministic time $T(n)^{c}$ for some large constant $c \in \mathbb{N}$. In other words, they showed that when solving decision problems, randomness can be deterministically simulated with a polynomial runtime overhead.

In a recent exciting work, Doron, Moshkovitz, Oh, and Zuckerman [11] asked if an even faster derandomization is possible: Could we prove that derandomization with a small polynomial overhead (i.e., derandomization in time $T(n)^{c}$ for a small value of c) follows from plausible hypotheses? Taken to the extreme, could it be that randomized algorithms for decision problems can be deterministically simulated with almost no runtime overhead? Their main result is that derandomization with only a quadratic time overhead (i.e., $c \approx 2$ ) is possible under a certain lower bound hypothesis; specifically, this conclusion follows from the hypothesis that $\mathcal{D T} \mathcal{I} \mathcal{M E}\left[2^{n}\right]$ is hard for randomized and non-deterministic circuits of very large size $\approx 2^{\cdot 99 \cdot n}$. That is:

THEOREM 1.1 (DERANDOMIZATION WITH NEAR-QUADRATIC OVERHEAD [11]). For every $\epsilon>0$ there exists $\delta>0$ such that the following holds. Assume that there exists $L \in \mathcal{D T} \mathcal{I} \mathcal{M E}\left[2^{n}\right]$ that cannot be computed by randomized SVN circuits ${ }^{1}$ of size $2^{(1-\delta) \cdot n}$, even infinitely-often. Then, for every time-constructible $T(n) \geq n$ we have that $\operatorname{pr} \mathcal{B P} \mathcal{T} \mathcal{I} \mathcal{M}[T(n)] \subseteq \operatorname{prDT} \mathcal{I} \mathcal{M E}\left[T(n)^{2+\epsilon}\right]$.

The result of Doron et al. [11] provides evidence that extremely fast derandomization might be possible, and moreover opens the door to asking if an even faster derandomization, namely with overhead $\approx T(n)^{1.01}$, might also be possible. However, the hypothesis

${ }^{1}$ Randomized SVN circuits are the non-uniform analogue of $\mathcal{M} \mathcal{A} \cap \operatorname{co} \mathcal{M} \mathcal{A}$ 
in Theorem 1.1 is considerably stronger than the hypotheses in the classical works: It refers to circuits that are not only larger (i.e., of size $2^{.99 \cdot n}$ rather than $2^{.01 \cdot n}$ ), but that also use randomness and non-determinism; that is, it refers to a lower bound for the non-uniform analogue of $\mathcal{M} \mathcal{A} \cap \operatorname{co} \mathcal{M} \mathcal{A}$. Needless to say, these additional resources might significantly increase the power of nonuniform circuits. ${ }^{2}$ Moreover, in contrast to classical "hardness-torandomness" works, the hypothesis in Theorem 1.1 is not known to be necessary for the existence of the corresponding PRG that they construct.

\subsection{Our Contributions: Bird's Eye}

The current paper extends the line of inquiry opened by [11], focusing on the possibility of extremely fast derandomization, and provides answers to several open questions that arose from their work. We now describe our results informally and in high-level, and later on we will elaborate in more detail.

1. Simulating randomness with very small overhead. The main open question following Theorem 1.1 is whether or not we can derandomize probabilistic algorithms in time that is close to linear $T(n)^{1+\epsilon}$, rather than in quadratic time $T(n)^{2+\epsilon}$.

We provide an affirmative answer to this question, conditioned on a plausible hypothesis, which is formally incomparable to the one in Theorem 1.1 but is arguably more standard. Specifically, we show that probabilistic algorithms running in time $T(n)$ can be deterministically simulated in time $n \cdot T(n)^{1+\epsilon}$, conditioned on the following: There exist one-way functions secure against polynomial-sized circuits, and there exists a problem in time $2^{k \cdot n}$ that is hard for algorithms that run in time $2^{(k-.01) \cdot n}$ and use $2^{.99 n}$ bits of non-uniform advice, where $k$ is a sufficiently large constant (see Theorem 1.2, and see the subsequent discussion for a comparison with Theorem 1.1).

The second assumption may be viewed as a stronger version of the classical time-hierarchy theorem, asserting that the timehierarchy holds even when the "weaker" class is given a nearmaximal amount of non-uniform advice. This assumption (or, more accurately, a relaxation of it that we use) is essentially necessary to obtain the derandomization conclusion using PRGs (see Section 1.2.2), and is a natural extension of hardness hypotheses from classical "hardness-to-randomness" results.

2. Optimizing the time overhead. We further improve the derandomization time to $n^{1+\epsilon} \cdot T(n)$ for natural special cases, conditioned on hypotheses that are slightly more technically involved and/or mildly stronger (see Theorem 1.4). This time bound almost matches the straightforward non-uniform derandomization, and we prove that it is essentially optimal, under a "counting" version of the non-deterministic strong exponential time hypothesis (i.e., under \#NSETH; see Theorem 1.3).

3. Average-case derandomization with almost no overhead. Bypassing the conditional lower bound in Theorem 1.3, we show a faster derandomization for polynomial-time algorithms that succeeds on average case, rather than in the worst-case. Specifically,

${ }^{2}$ For a recent demonstration of the power of $\mathcal{M} \mathcal{A}$ algorithms that run in exponential time, see [29]; we refer the reader to the corresponding discussion in [11, Section 1.6]. our derandomization algorithm runs in time $n^{\epsilon} \cdot T(n)$, and succeeds with probability $1-n^{-\omega(1)}$ with respect to all distributions samplable in time $T(n)$ (see Theorem 1.7).

4. Fast derandomization via simple proof paradigms. The proof of Theorem 1.1 in [11] is highly non-trivial, relying on refined technical notions and on complicated analyses. In contrast, all of our results rely on simple and intuitive proofs that use only standard technical tools. In particular, the results mentioned above rely on proof strategies that are significantly different than the ones in [11].

We also present a proof for Theorem 1.1 that is simpler than the one in [11] and relies on an observation of independent interest: Any PRG construction that is analyzed as "extracting randomness from a pseudoentropic string" (in particular, the construction of [11]) can be analyzed via a different proof strategy that is both simpler and more general (see Section 1.4). As one application, we extend Theorem 1.1 by showing that derandomization with either cubic or quartic overhead is possible, under hardness assumptions that are similar to the one in Theorem 1.1, yet refer only to SVN circuits that do not use randomness (see Theorem 1.8).

\subsection{Worst-Case Derandomization with Very Small Overhead}

Our first result is that under a plausible hardness hypothesis, probabilistic algorithms that run in time $T(n)$ can be deterministically simulated in time $n \cdot T(n)^{1+\epsilon}$, for an arbitrarily small constant $\epsilon>0$ and for all time bounds $T(n)$.

Similarly to other results in the "hardness-to-randomness" lineof-works, our derandomization relies on a PRG construction. However, the standard approach of constructing a PRG that "fools" nonuniform circuits of size $T(n)$ cannot work here, because such a PRG requires a seed of length at least $\log (T(n))$ (and evaluating a time- $T$ algorithm at each output of the PRG requires time $\left.T(n)^{2}\right)$. Our way to bypass this obstacle is to observe that the standard approach is an "overkill": When transforming a probabilistic algorithm into a distinguisher for the PRG, the distinguisher runs in time $T(n)$ but only uses $n$ bits of non-uniform advice (i.e., the advice corresponds to the input, which is of length $n$ rather than $T(n))$. Thus, it suffices to "fool" the foregoing class of distinguishers, and indeed there exists a non-explicit PRG for this class with sub-logarithmic seed length; that is, the distinguisher class can be modeled by $\mathcal{D T} \mathcal{I} M \mathcal{E}[N] / T^{-1}(N)$ (where the notation alludes to $N=T(n)$ ), and it can be "fooled" by a (non-explicit) PRG with seed length $(1+o(1)) \cdot \log \left(T^{-1}(N)\right) .^{3}$

In our first result we construct a PRG that yields derandomization in time $n \cdot T(n)^{1+\epsilon}$, conditioned on the following. First, we assume that there exist non-uniformly secure one-way functions; recall that this assumption is not known to imply (by itself) derandomization in less than sub-exponential time. Secondly, we assume that there exists a problem decidable in time $2^{k \cdot n}$ that cannot be solved in time $2^{(k-\delta) \cdot n}$ with $2^{(1-\delta) \cdot n}$ bits of non-uniform advice, where $\delta$ is sufficiently small and $k$ is sufficiently large. As mentioned above, the latter hypothesis can be interpreted as a strengthening

\footnotetext{
${ }^{3}$ To see this, for every input length $N$ we "fool" the first $\epsilon(N)$ machines that run in time $N$, instantiated with every possible advice, where $\epsilon$ is any super-constant function. This yields at most $\epsilon(N) \cdot 2^{T^{-1}(N)}$ distinguishers, and therefore a seed of length $\log \left(T^{-1}(N)\right)+\log (\epsilon(N))$ suffices.
} 
of the classical time-hierarchy theorem, since it asserts that (loosely speaking) there are problems solvable in time $T(n)=2^{k \cdot n}$ that cannot be solved in time slightly smaller than $T(n)$ even with a near-maximal amount of non-uniform advice.

THEOREM 1.2 (DERANDOMIZATION WITH VERY SMALL OVERHEAD FOR ALL PROBABILISTIC ALgORITHMS). For every $\epsilon>0$ there exists $\delta>0$ such that the following holds. Let $T: \mathbb{N} \rightarrow \mathbb{N}$ be any timeconstructible non-decreasing function, and let $k=k_{\epsilon, T} \geq 1$ be a sufficiently large constant. ${ }^{4}$ Assume that there exist one-way functions that are secure against polynomial-sized circuits, and that there exists $L \in$ $\mathcal{D T} \mathcal{I} \mathcal{M E}\left[2^{k \cdot n}\right]$ such that $L \notin$ i.o. $\mathcal{D} \mathcal{T} \mathcal{I} \mathcal{M}\left[2^{(k-\delta) \cdot n}\right] / 2^{(1-\delta) \cdot n}$. Then $\operatorname{pr} \mathcal{B P} \mathcal{T} \mathcal{I} \mathcal{M}[T(n)] \subseteq \operatorname{prDT} \mathcal{I} \mathcal{M E}\left[n \cdot T(n)^{1+\epsilon}\right]$.

The non-cryptographic hypothesis in Theorem 1.2 can be relaxed, to only require that the amortized time-complexity of $L$ when printing its entire truth-table will be $2^{k \cdot n}$ (rather than requiring each entry in the truth-table to be computable in such time; see [ 8 , Theorem 4.10]). The reason that we mention this relaxation is that the existence of such an $L$ is necessary for the PRG conclusion (see Proposition 1.6 and [8, Theorem 4.11]). This relaxation will apply to all results in the current section, but for simplicity we will avoid mentioning it explicitly, and defer the full result statements to the technical section.

Thus, one of our assumptions in Theorem 1.2 is necessary for the conclusion, and the other is a standard and fundamental one. Moreover, both assumptions refer only to standard computational models that do not use non-determinism or randomness (rather than to non-uniform analogues of $\mathcal{M} \mathcal{A} \cap \operatorname{co} \mathcal{M} \mathcal{A}$ ). Thus, we argue that our hypothesis is more standard and appealing than the one in Theorem 1.1. Intuitively, the main part in our hypothesis that is stronger (and allows for faster derandomization) is that our hardness assumption refers to a separation of uniform algorithms from non-uniform procedures in a "higher" time bound (i.e., in time $2^{k \cdot n}$ rather than in time $2^{n}$ ).

Derandomizing "better-than-brute-force" algorithms. The benefit in derandomization as in Theorem 1.2 (compared to, say, derandomization in quadratic time) is particularly salient when considering randomized algorithms that run in time close to that of a "bruteforce" algorithm. Many such "better-than-brute-force" randomized algorithms are known, for example for $\mathcal{N} \mathcal{P}$-complete graph problems (see, e.g., $[4,10]$ ), for satisfiability of formulas and circuits (see, e.g., $[9,20]$ ), and for $\mathcal{N} \mathcal{P}$-complete algebraic problems (see, e.g., [17]). For all these problems, derandomization in time $T(n)^{1+\epsilon}$ would yield a better-than-brute-force deterministic algorithm, but derandomization in time $T(n)^{2}$ is trivial. $^{5}$

Derandomization as in Theorem 1.2 also implies that lower bounds for deterministic algorithms yield near-identical lower bounds for randomized algorithms: For example, if for every $\epsilon>0$ the hypothesis of Theorem 1.2 holds, then the Strong ExponentialTime Hypothesis (SETH) is equivalent to its randomized version (i.e., to rSETH).

${ }^{4}$ When $T$ is a polynomial the constant $k$ will be very close to the polynomial power of $T$, and when $T$ is super-polynomial the constant $k$ will be linear in $1 / \epsilon$; see $[8$, Theorem 4.10].

${ }^{5}$ For incomparable results regarding derandomization of slow probabilistic algorithms, see [2].
1.2.1 Can We Further Reduce the Overhead? The derandomization time overhead of $T(n) \mapsto n \cdot T(n)^{1+\epsilon}$ is small, but is it as small as it can be? We prove several additional results that address this question, both by improving the upper-bound and by showing a near-matching conditional lower bound.

Recall that the known non-uniform derandomization of $\operatorname{pr} \mathcal{B P} \mathcal{T} \mathcal{I} \mathcal{M E}[T]$ yields circuits of size $O(n \cdot T(n)) .{ }^{6}$ We first show that such an overhead is unavoidable, at least for uniform algorithms, ${ }^{7}$ conditioned on a counting version of the Non-Deterministic Strong Exponential-Time Hypothesis (NSETH), which is weaker than NSETH itself. Specifically, extending a result of Williams [29], we prove that derandomization in time $O(n \cdot T(n))$ is optimal if for every $\epsilon>0$ there does not exist a non-deterministic machine that counts the number of satisfying assignments of a $k$-SAT formula over $n$ bits in time $2^{(1-\epsilon) \cdot n}$, assuming that $k=k_{\epsilon}$ is sufficiently large (see [8, Section 6]).

THEOREM 1.3 (CONDITIONAL NECESSITY OF THE MULTIPLICATIVE OVERHEAD OF $n$ ). Assuming\#NSETH we have that $\mathcal{B P} \mathcal{T} \mathcal{I} M \mathcal{E}[T] \nsubseteq$ $\mathcal{D T} \mathcal{I} \mathcal{M E}\left[n^{1-\epsilon} \cdot T(n)\right]$, for every polynomial $T$ and $\epsilon>0$.

Matching Theorem 1.3, we improve the derandomization overhead in Theorem 1.2 to $n^{1+\epsilon} \cdot T(n)$ for a large class of probabilistic algorithms, under hypotheses similar to the ones in Theorem 1.2. We state the following result in a slightly suboptimal way for simplicity, and mention afterwards how it can be improved.

THEOREM 1.4 (DERANDOMIZATION WITH NEAR-OPTIMAL OVERHEAD; INFORMAL). For every $\epsilon>0$ there exists $\delta>0$ such that for every "nice" $T(n) \leq 2^{n^{o(1)}}$ the following holds. Assume that for some $\gamma>0$ there exist one-way functions that are secure against circuits of size $2^{n^{\gamma}}$, and that there exists $L \in \mathcal{D T} \mathcal{I} \mathcal{M E}\left[2^{\delta \cdot n} \cdot T^{\prime}(n)\right]$ such that $L \notin$ i.o.D $\mathcal{D} \mathcal{I} \mathcal{M} E\left[T^{\prime}\right] / 2^{(1-\delta) \cdot n}$, where $T^{\prime}(n)=2^{O(\delta \cdot n)}$. $T\left(2^{(1-\delta) \cdot n}\right)$. Then, $\operatorname{pr} \mathcal{B P T} \mathcal{I} \mathcal{M E}[T] \subseteq \operatorname{pr} \mathcal{D T} \mathcal{I} \mathcal{M E}\left[n^{1+\epsilon} \cdot T(n)\right]$.

The hypothesis in Theorem 1.4 can be improved for polynomial time functions $T(n)=\operatorname{poly}(n)$. Specifically, in this case we only need the hypothesized one-way function to be secure against polynomial-sized circuits (see [8, Theorem 4.8] for details).

1.2.2 Batch-Computable PRGs and Problems with Bounded Amortized Complexity. Recall that the results in this section are obtained by constructing PRGs for the class $\mathcal{D T} \mathcal{I} \mathcal{M}[O(N)] / T^{-1}(N)$, which (ideally) have seed length $\ell \approx \log \left(T^{-1}(N)\right)=\log (n)$. Also recall that our goal is to obtain derandomization in time $n^{1+\epsilon} \cdot N$.

Unlike classical results, we do not prove that our PRGs are computable in time close to $N$ on each of the $2^{\ell}$ seeds, but rather only "batch-computable" in time close to $2^{\ell} \cdot N$ on all seeds at once. Indeed, such PRGs still suffice for the standard derandomization approach of enumerating over all seeds. And moreover, as mentioned after Theorem 1.2, the "batch-computable" PRGs that we construct also follow from the relaxed hypothesis that asserts an upper-bound

\footnotetext{
${ }^{6}$ This is since for every probabilistic algorithm and $n \in \mathbb{N}$, by a Chernoff bound there exist $O(n)$ fixed random strings that lead the algorithm to a correct decision on all inputs.

${ }^{7}$ Note that many problems can be solved in constant probabilistic time $T(n)=O(1)$ but require linear deterministic time $O(n)$ (e.g., estimating the Hamming weight of the input). Nevertheless, the lower bound for this specific time bound does not rule out an additive derandomization overhead of $n$.
} 
on the amortized time-complexity of the hard problem when computing its entire truth-table (rather than a worst-case time bound); and the existence of such a problem is in fact necessary to get "batch-computable" PRGs.

The point is that the relationship that we show between the latter two objects is quantitatively tighter than the known relationship between standard PRGs (that are efficiently-computable on each seed) and hard problems (with bounded worst-case complexity). Thus, in the context of derandomization with almost no time overhead, it turns out to be more fruitful to study the two weaker objects. To be more explicit about this point, let us state a special case of the connection between these two objects. (The result follows from the technical versions of results that were already stated or mentioned above, but its parametrization is less clean since we wish to highlight the parametric tightness.)

Definition 1.5 (amortized time complexity). For $f:\{0,1\}^{*} \rightarrow$ $\{0,1\}$, we say that $f \in$ amort-DTI $\mathcal{I} \mathcal{E}[T]$ if for every $n \in \mathbb{N}$, the truth-table of $f$ on $n$-bit inputs can be printed in time $2^{n} \cdot T(n)$.

Proposition 1.6 (NEAR-EQUIVALENCE BETWEEN BATCH-COMPUTABLE PRGS AND PROBLEMS WITH BOUNDED AMORTIZED TIME COMPLEXITY, THE POLYNOMIAL SETTING). There exists a universal constant $c>1$ such that the following holds. Assume there exists one-way functions that are secure against polynomial-sized circuits. Then, for every $\epsilon>0$ there exist $\delta, \delta^{\prime}>0$ such that for any fixed constant $k \geq 1$ :

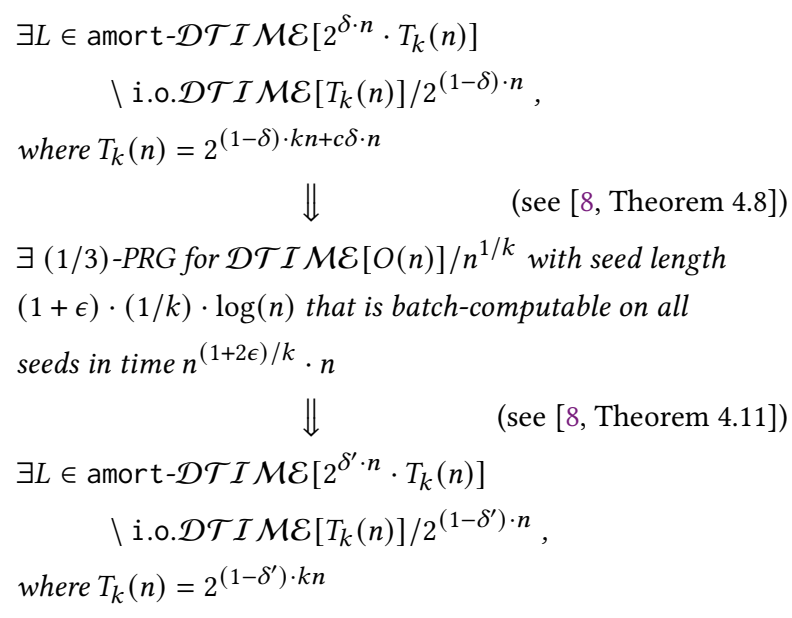

\subsection{Average-Case Derandomization with Almost No Overhead}

Bypassing the conditional lower bound in Theorem 1.3, we show that a faster derandomization is possible if we are willing to settle for derandomization that succeeds only on most inputs, rather than in worst-case. Recall that for any $L \in \mathcal{B P T} \mathcal{I} \mathcal{M E}[T]$ and any distribution $\mathcal{D}$ over $\{0,1\}^{n}$ there exists a circuit family of size $O(T(n))$ that correctly decides $L_{n}$ with high probability over choice of input from $\mathcal{D}$ (say, with probability 0.99 ). Moreover, when we restrict the class of distributions only to those that are samplable in time $T(n)$, then there exists a single circuit family of size $\tilde{O}(T(n))$ that correctly decides $L$ with high probability with respect to all distributions in this class. ${ }^{8}$ (Note that this class of distributions is quite natural, and in particular contains the uniform distribution.)

We show a near-matching explicit (i.e., uniform) derandomization under a hypothesis that is similar to the one in Theorem 1.2. Specifically, under this hypothesis, for every $L \in \mathcal{B P T} \mathcal{I} \mathcal{M}[T]$ we construct a deterministic algorithm $A_{L}$ that runs in time $n^{\epsilon} \cdot T(n)$ and succeeds with high probability with respect to every $T$-time samplable distribution. (In particular, with respect to uniform distribution.) We will focus on the setting of polynomial time bounds $T(n)=n^{k}$, since this is the more interesting setting for improving the worst-case bound of $n^{1+\epsilon} \cdot T(n)$ to the average-case bound $n^{\epsilon} \cdot T(n)$.

THEOREM 1.7 (AVERAGE-CASE DERANDOMIZATION WITH OVERHEAD $\left.n^{\epsilon}\right)$. For every $\epsilon>0$ there exists $\delta>0$ such that for every $T(n)=n^{k}$ the following holds. Assume that there exist one-way functions secure against polynomial-sized circuits, and that there exists $L_{0} \in \mathcal{D T} \mathcal{I} \mathcal{M E}\left[2^{\delta \cdot n} \cdot T^{\prime}(n)\right] \backslash$ i.o.D $\mathcal{D} \mathcal{I} \mathcal{M E}\left[T^{\prime}\right] / 2^{(1-\delta) \cdot n+1}$, where $T^{\prime}(n)=2^{(1-\delta) \cdot(2 k / \epsilon) \cdot n+O(\delta \cdot n)}$. Then, for every $L \in \mathcal{B P T} \mathcal{I} \mathcal{M} \mathcal{E}[T]$ there exists a deterministic algorithm $A_{L}$ that runs in time $n^{\epsilon} \cdot T(n)$ such that for every $T$-time samplable distribution $\mathcal{D}$ it holds that $\operatorname{Pr}_{x \sim \mathcal{D}_{n}}\left[A_{L}(x)=L(x)\right]=1-n^{-\omega(1)}$.

An appealing interpretation for the derandomization in Theorem 1.7 is that there exists a deterministic algorithm $A_{L}$ such that every $T$-time algorithm that tries to find an input $x$ such that $A_{L}(x) \neq L(x)$ succeeds only with negligible probability.

\subsection{Fast Derandomization Via a Simple Paradigm}

The starting point for our other contributions is an alternative and considerably simpler proof for Theorem 1.1. Using a different high-level proof strategy, we rely on the hypothesis to construct a very simple PRG, and analyze it in a way that avoids essentially all of the involved technical work that was carried out in [11]. This alternative proof leads us to two further contributions:

(1) Our simple proof is flexible, and allows us to extend the original result in several directions. In particular, we can relax the hardness hypothesis while settling on derandomization with cubic or quartic overhead (see below).

(2) Our proof strategy simplifies a well-known proof strategy for PRG constructions in general. Specifically, we show that any PRG construction that relies on "extracting randomness from a pseudoentropic string” (as in [11], following [3, 12, 14]) can be analyzed in a simpler way.

The details of our alternative proof strategy are presented in Section 2.2. In a gist, given a construction $G\left(s_{0}, s_{1}\right)=\operatorname{Ext}\left(G_{0}\left(s_{0}\right), s_{1}\right)$ that is analyzed as "extracting randomness from a pseudoentropic string", we show that $G$ can be analyzed in the following way: We first show that Ext reduces the derandomization problem to the problem of quantified derandomization (via a non-standard reduction); and then we show that $G_{0}$ solves the latter problem,

\footnotetext{
${ }^{8}$ To see this, for every $n \in \mathbb{N}$, consider the first $n$ Turing machines that run in time $T$. By a Chernoff bound, there exist $O(\log (n))$ random strings that lead the probabilistic algorithm to be correct with probability 0.99 on each of the $n$ distributions sampled by the $n$ Turing machines. The average-case error (of 0.99) can be decreased by first applying error-reduction to the original probabilistic machine.
} 
concluding that $G$ is a PRG. This analysis follows a classical idea of Sipser [23], which was recently highlighted in [13] and in a sequence of follow-up works concerning quantified derandomization. We also argue that this simpler analysis applies to a potentiallylarger class of constructions (see Section 2.2).

As mentioned above, our proof allows us to mildly relax the hypothesis of Theorem 1.1 while deducing an only mildly slower derandomization. Specifically, we show that the hypothesis in Theorem 1.1 can be relaxed to refer only to SVN circuits, which are non-uniform analogues of $\mathcal{N P} \cap \operatorname{coN} \mathcal{P}$, while deducing derandomization with either cubic overhead $T(n)^{3+\epsilon}$ or quartic overhead $\approx T(n)^{4+\epsilon}$ (rather than quadratic overhead), depending on the specific hardness hypothesis. In more detail:

THEOREM 1.8 (FAST DERANDOMIZATION FROM HARDNESS FOR SVN CIRCuITs). For every $\epsilon>0$ there exists $\delta>0$ such that the following holds.

(1) Assume that there exists $L \subseteq\{0,1\}^{*}$ whose entire truth-table on $n$-bit inputs can be printed in time $2^{(3 / 2) \cdot n}$, but that cannot be computed (on an input-by-input basis) by SVN circuits of size $2^{(1-\delta) \cdot n}$, even infinitely-often. Then, for every timeconstructible $T: \mathbb{N} \rightarrow \mathbb{N}$ we have that $\operatorname{pr} \mathcal{B P} \mathcal{I} \mathcal{I} \mathcal{M E}[T(n)] \subseteq$ $\operatorname{pr} \mathcal{D} \mathcal{T} \mathcal{I} \mathcal{M E}\left[T(n)^{3+\epsilon}\right]$.

(2) Assume that there exists $L \in \mathcal{D T} \mathcal{I} M \mathcal{E}\left[2^{n}\right]$ such that $L$ cannot be computed by $S V N$ circuits of size $2^{(1-\delta) \cdot n}$, even infinitely-often. Then, for every time-constructible $T: \mathbb{N} \rightarrow \mathbb{N}$ we have that $\operatorname{pr} \mathcal{B P} \mathcal{T} \mathcal{I} \mathcal{M E}[T(n)] \subseteq \operatorname{prDT} \mathcal{I} \mathcal{M E}\left[T(n)^{4+\epsilon}\right]$.

We also construct a near-optimal algorithm for a natural setting of quantified derandomization, under a plausible hypothesis that refers to hardness for SVN circuits. Loosely speaking, we show that randomized time- $T$ algorithms that err on at most $2^{T(n)^{.99}}$ of their random choices can be deterministically simulated in time $T(n)^{1.01}$, if there exists a function whose truth-table on $n$-bit inputs can be printed in time $2^{(1.01) \cdot n}$, but that is hard to compute (on an input-by-input basis) for SVN circuits of size $2^{99 \cdot n}$. See [8, Theorem 5.4] for details.

New light on bypassing the hybrid argument. A well-known challenge in proving results such as the ones in Theorems 1.1 and 1.2 is that the analysis of the PRG construction needs to avoid a certain hybrid argument (for a detailed explanation see [3, Sec. 1.2], and also [12]). Indeed, the proofs in $[3,11]$ as well as our proofs avoid such an argument. However, we believe that the observations in the current section allow to better understand how this barrier was bypassed in all these works, which sheds new light on this challenge. Further details appear in Section 2.2.

\section{PROOF OVERVIEWS}

Throughout the section we will be somewhat informal with respect to the precise values of parameters, and in particular denote by $\epsilon>0$ an unspecified constant that should be thought of as arbitrarily small. Also, for simplicity we explain how to construct PRGs with constant error; the extensions to PRGs with error $n^{-.01}$ are straightforward.

\subsection{Proof of Theorems 1.2, 1.4 and 1.7}

Let us first see what is the core difficulty that yields a large polynomial overhead in existing "hardness-to-randomness" proofs. Recall that for a probabilistic algorithm $M$ with running time $T(n)$ and a fixed input $x \in\{0,1\}^{n}$, we consider a distinguisher $D_{x}$ that gets as input random coins $r \in\{0,1\}^{N}$, where $N=T(n)$, runs in time $N$, and outputs the decision of $M$ at input $x$ with randomness $r$. If we can construct a PRG that "fools" all potential distinguishers $D_{x}$, then we can use this PRG to derandomize $M$.

Classical "hardness-to-randomness" proofs (following [19]) rely on reconstructive PRGs. Such a PRG is an oracle machine $G$ that gets a random seed and access to a function $f$ and satisfies the following: Any efficient procedure $D$ that distinguishes the output distribution of $G^{f}$ from the uniform distribution can be transformed to an efficient procedure $C_{f}$ that computes $f$. As a contrapositive, if $f$ is hard for every efficient procedure, then $G^{f}$ "fools" every potential efficient distinguisher $D$.

The key bottleneck in the reconstructive proof approach is the overhead in transforming $D$ into $C_{f}$. In the state-of-the-art construction of Umans [28] (following [15, 19, 22, 24, 25]), the transformation overhead is a large polynomial, say $\left|C_{f}\right|=|D|^{c}=N^{c}$. Thus, we must assume that $f$ is hard for circuits of size $N^{c}$, which means that the time it takes to compute $G^{f}$ is larger than $N^{c}=T(n)^{c}$. This is where the large derandomization overhead comes from, and this is what we want to avoid.

The first idea: Composing two "low-cost" PRGs. We will construct a PRG with optimal seed length $(1+\epsilon) \cdot \log (N)$ and running time $N^{1+\epsilon}$ by composing two suboptimal yet "low-cost" PRGs; that is, each of the two PRGs falls short of achieving the parameters we need by itself, but is nevertheless computable in time $N^{1+\epsilon}$. The first PRG will have short seed length $(1+\epsilon) \cdot \log (N)$ but also short output length $N^{\epsilon}$; and the second PRG will have a relatively-long seed $N^{\epsilon}$ but a sufficient output length $N$.

The first PRG follows from the observation that the transformation of hardness into randomness entails very little overhead when the PRG outputs a relatively-short string. In fact, for this purpose we can even use an instantiation of the classical construction of Nisan and Wigderson [19] (with variations a-la [21]): Using a function $f$ whose truth-table is of size $|f|=N^{1+O(\epsilon)}$, a suitable instantiation of the NW PRG outputs $M=N^{\epsilon}$ pseudorandom bits using seed length $(1+O(\epsilon)) \cdot \log (N)$, in time $N^{1+O(\epsilon)}$, and with a small reconstruction overhead such that $\left|C_{f}\right|=|f|^{\epsilon} \cdot|D|+|f|^{1-2 \epsilon}<|f|^{1-\epsilon}$ (i.e., the reconstruction procedure is an oracle machine that gets access to $D$, runs in time $|f|^{\epsilon}$, and uses $|f|^{1-2 \epsilon}$ bits of advice; see [8, Theorem 4.1]). ${ }^{9}$ Thus, if $f$ is hard for circuits of size $|f|^{1-\epsilon}$, then the PRG "fools" $D$.

The observation underlying the second PRG is that standard cryptographic assumptions yield a very fast PRG with polynomial stretch. Specifically, assuming the existence of a one-way function that is secure against polynomial-sized circuits, there exists a PRG $G^{\text {cry }}$ with stretch $M \mapsto N$ that can be computed in time $N^{1+\epsilon}$ and "fools" any distinguisher of size $N$ (see [8, Section 4.1]). The

${ }^{9}$ The parameters stated here are not fully accurate (e.g., we need $M=N^{\Omega\left(\epsilon^{2}\right)}$ for this to be true), but the difference is immaterial for this high-level discussion. See [8, Section 4] for the full details. 
composed PRG will take a seed $w \in\{0,1\}^{(1+O(\epsilon)) \cdot \log (N)}$, map it to $N W(w) \in\{0,1\}^{M}$, and output $G(w)=G^{\text {cry }}(N W(w)) \in\{0,1\}^{N}$ The composition of $G^{\text {cry }}$ and of $N W$ indeed "fools" $D$, where the crucial point is that applying the "outer" PRG $G^{\text {cry }}$ can be thought of as yielding a distinguisher $D^{\prime}=D \circ G^{\text {cry }}$ of approximately the same size as $D$ (and therefore $N W$ "fools" $\left.D^{\prime}\right) .{ }^{10}$

This argument yields derandomization with quadratic overhead; that is, with running time $N^{2+O(\epsilon)}=T(n)^{2+O(\epsilon)}$. This is because we will evaluate $D$, which is of size $N$, on each of the $N^{1+O(\epsilon)}$ outputs of the PRG. Thus, so far we deduced the conclusion of Theorem 1.1 from incomparable hypotheses (which refer only to standard circuits), but still fall short of proving the stronger conclusion of Theorem 1.2.

The second idea: Using small and extremely hard truth-tables to fool distinguishers with "a little" non-uniformity. The bottleneck in the argument above is that the seed length of the NW PRG is $(1+O(\epsilon)) \cdot \log (N)$. In general, the seed length of the NW PRG is proportional to the truth-table size of the hard function, and in our instantiation of the NW PRG the seed length is $(1+\epsilon) \cdot \log (|f|)$. However, it a-priori seems impossible to use a smaller truth-table, since our distinguisher is a non-uniform circuit of size $N$, and we cannot assume hardness of a function with truth-table size $|f|<N$ for circuits of size $N$ (i.e., it is trivial to compute such a function with $N$ bits of advice).

As mentioned in Section 1, the pivotal observation to solve this problem is that when the distinguisher $D=D_{x}$ models the execution of a probabilistic algorithm with running-time $N=T(n)$ on an input $x$, then $D$ can be thought of as a time- $N$ algorithm that uses only $|x|=n=T^{-1}(N)$ bits of non-uniform advice. In other words, to derandomize time- $T$ algorithms it suffices to "fool" the class $\mathcal{D T} \mathcal{I} \mathcal{M E}[N] / T^{-1}$, rather than all circuits of size $N$. This opens the door to instantiating the PRG using a very hard function whose truth-table is of size significantly smaller than the running time of our distinguisher; in other words, we will be using a function with super-exponential time complexity.

Let us spell out the resulting argument, and for concreteness let us focus on the setting of $T(n)=n^{k}$ for some constant $k$. The outer PRG $G^{\text {cry }}$ will have stretch $n^{\epsilon} \mapsto n^{k}$, and the inner PRG NW will use a function of truth-table size $|f|=n^{1+O(\epsilon)}$ and a seed of length $(1+O(\epsilon)) \cdot \log (n)$ to output $n^{\epsilon}$ bits. Recall that the reconstruction procedure of NW is an oracle machine that runs in time $|f|^{\epsilon}$ and uses $|f|^{1-2 \epsilon}$ bits of non-uniform advice; thus, a distinguisher that runs in time $T(n)$ and uses $n$ bits of advice yields an algorithm $A_{f}$ for $f$ that runs in time $T(n) \cdot n^{O(\epsilon)}$ and uses $n+|f|^{1-2 \epsilon}<|f|^{1-\epsilon}$ bits of advice (see [8, Theorem 4.1]). When we consider the complexity of $A_{f}$ as a function of the input size $\ell=\log (|f|)$ to $f$, we get an algorithm that runs in time $T\left(2^{(1-O(\epsilon)) \cdot \ell}\right) \cdot 2_{2}^{O(\epsilon \cdot \ell)}$ and uses

\footnotetext{
${ }^{10}$ To see this, observe that $\operatorname{Pr}\left[D^{\prime}\left(N W\left(\mathbf{u}_{\ell}\right)\right)=1\right]=\operatorname{Pr}\left[D\left(G^{\text {cry }}\left(N W\left(\mathbf{u}_{\ell}\right)\right)\right)=1\right]$, where $\ell=(1+O(\epsilon)) \cdot \log (N)$. Since $D^{\prime}$ is of approximately the same size as $D$, we can instantiate the NW PRG with parameters very similar to the ones above, and assuming that $f$ is hard for circuits of size $|f|^{1-\epsilon}$, the distribution $N W\left(\mathbf{u}_{\ell}\right)$ is pseudorandom for $D^{\prime}$. Since $\operatorname{Pr}\left[D^{\prime}\left(\mathbf{u}_{M}\right)=1\right] \approx \operatorname{Pr}\left[D\left(\mathbf{u}_{N}\right)=1\right]$, the distribution $G^{\text {cry }}\left(N W\left(\mathbf{u}_{\ell}\right)\right)$ is pseudorandom for $D$.
}

$2^{(1-\epsilon) \cdot \ell+1}$ bits of advice. Thus, for this to work we need to assume that $f \notin$ i.o. $\mathcal{D T} \mathcal{I} \mathcal{M} E\left[T\left(2^{(1-\epsilon) \cdot \ell}\right) \cdot 2^{O(\epsilon \cdot \ell)}\right] / 2^{(1-\epsilon) \cdot \ell+1} .11$

The last necessary missing piece: Batch-computable PRGs and hard functions with bounded amortized time complexity. The resulting derandomization algorithm computes the output-set of the PRG, and evaluates $D$ at each of the strings in this set. Now, recall that we want this algorithm to run in time $n^{1+O(\epsilon)} \cdot T(n)$. It is not clear if we can compute the PRG at each seed in time close to $T(n)$ (as is the standard approach); nevertheless, we can still compute the entire output-set of the PRG "in a batch" in time $n^{1+O(\epsilon)} \cdot T(n)$, which suffices for our purposes.

For concreteness, let us still focus on the setting of $T(n)=n^{k}$. We first compute the entire truth-table of $f$, and we need to do so in time at most $n^{1+O(\epsilon)} \cdot T(n)=2^{\ell} \cdot 2^{O(\epsilon \cdot \ell)} \cdot T\left(2^{(1-O(\epsilon)) \cdot \ell}\right)$. Thus, we have to assume that when computing the entire truth-table of $f$, the amortized time cost per entry is no more than $2^{O(\epsilon \cdot \ell)}$. $T\left(2^{(1-O(\epsilon)) \cdot \ell}\right)$. (Note that the constant hidden inside the $O$-notation in $2^{O(\epsilon \cdot \ell)}$ may be larger than the constant hidden inside the $O$ notation in $2^{O(\epsilon \cdot \ell)}$ in our lower bound.) As mentioned in Section 1, the existence of such a function is necessary in order to construct a "batch-computable" PRG as the one that we are constructing (see [8, Theorem 4.11]). Now, given access to $f$, we can compute the output-set of the NW PRG in time $n^{1+O(\epsilon)}$ (i.e., we compute the combinatorial designs once in advance, and similarly apply an error-correcting code to the truth-table of $f$ once in advance; see [8, Appendix A]). Finally, we evaluate $D^{\prime}=D \circ G^{\text {cry }}$ on each of the $n^{1+O(\epsilon)}$ resulting strings. The PRG $G^{\text {cry }}$ can be assumed to run in time $T(n) \cdot n^{\epsilon}$ (see [8, Section 4.1]), and thus our final running-time is indeed $n^{1+O(\epsilon)} \cdot T(n)$.

The above argument proves the special case of Theorem 1.2 for polynomial time functions $T(n)=n^{k}$. (In fact, it even proves the stronger result for this special case, in which the derandomization time is $n^{1+\epsilon} \cdot T(n)$.) In [8, Section 4] we explain how to extend the argument to super-polynomial time functions $T(n)=n^{\omega(1)}$. In a gist, we will either reduce the super-polynomial case to the polynomial case using a padding argument, which yields derandomization in time $n \cdot T(n)^{1+\epsilon}$; or rely on a stronger cryptographic hypothesis to obtain an outer PRG $G^{\text {cry }}$ with super-polynomial stretch, which yields derandomization in time $n^{1+\epsilon} \cdot T(n)$. See further details in [8, Section 4]

Proof of Theorem 1.7: Faster average-case derandomization. For simplicity, let us prove that we can derandomize algorithms that run in time $T(n)=n^{k}$ in time $n^{\epsilon} \cdot T(n)$ with respect to the uniform distribution (rather than any $T$-time samplable distribution).

Given a probabilistic machine $M$ running in time $T$, consider the machine $M^{\prime}$ that gets input $w \in\{0,1\}^{m}$, maps it to $x=G^{\text {cry }}(w) \in$ $\{0,1\}^{n}$ where $n=m^{1 / \epsilon}$, and outputs $M(x)$. We now instantiate Theorem 1.2 with the time function $T^{\prime}(m)=m^{k / \epsilon}$, which bounds the running time of $M^{\prime}$. Using appropriate hypotheses (to apply Theorem 1.2 with the function $T^{\prime}$ ), we deduce there exists a PRG $G$ with seed length $(1+\epsilon) \cdot \log (m)$ that is batch-computable in time $m^{1+\epsilon} \cdot m^{k / \epsilon}$ and can be used to replace the random coins of $M^{\prime}$.

\footnotetext{
${ }^{11}$ To see that this is consistent with the time bound stated in Theorem 1.2, plug-in $T(n)=n^{k}$ to the time bound to see that $T\left(2^{(1-\epsilon) \cdot \ell}\right) \cdot 2^{O(\epsilon \cdot \ell)}=2^{(1-\epsilon) \cdot k \ell+O(\epsilon \cdot \ell)}=$ $2^{(1-(1-O(1 / k)) \cdot \epsilon) \cdot k \ell}$
} 
Now, a key point to note is that $M^{\prime}$ only uses its random coins as random coins for $M$. Hence, we deduce that the PRG $G$ can be used to replace the random coins of $M$ on any input $x \in\{0,1\}^{n}$ in the output-set of $G^{\text {cry }}$. Note that as a function of $|x|=n$, the PRG has seed length $(1+\epsilon) \cdot \log \left(n^{\epsilon}\right)<2 \epsilon \cdot \log (n)$ and running time at most $n^{\epsilon(1+\epsilon)} \cdot n^{k}<n^{k+2 \epsilon}$.

Of course, this still doesn't mean that the $G$ can be used to replace the random coins of $M$ on inputs $x \in\{0,1\}^{n}$ that are not in the output-set of $G^{\text {cry }}$. The crucial observation is that we can now reuse the pseudorandomness properties of $G^{\text {cry }}$ to "fool" a second test (the first use of the pseudorandomness of $G^{\text {cry }}$ was in our instantiation of Theorem 1.2). Specifically, recall that $G^{\text {cry }}$ is a cryptographic PRG that was obtained relying on the existence of one-way functions, and hence it can be shown to "fool" circuits of arbitrary polynomial size (see [8, Proposition 4.3]). Now, consider an algorithm $T$ that gets input $x \in\{0,1\}^{n}$ and checks whether or not the pseudorandom coins produced by $G$ can be used to replace the random coins of $M$ at $x .{ }^{12}$ Since $T$ can be implemented by a circuit of size poly $\left(n^{k}\right)$, it is "fooled" by $G^{\text {cry }}$. And since for all $x$ in the output-set of $G^{\text {cry }}$ it holds that $T(x)=1$, it follows that for almost all $x \in\{0,1\}^{n}$ it holds that $T(x)=1$. Hence, for almost all $x \in\{0,1\}^{n}$ we can use $G$ to replace the random coins of $M$ at $x$.

The foregoing argument extends naturally to any $T$-time samplable distribution. For any $T$-time sampling algorithm $S$, we use $G^{\text {cry }}$ with stretch $m \mapsto T(n)$, and define $M^{\prime}(x)=M\left(S\left(G^{\text {cry }}(x)\right)\right)$ (this generalizes the foregoing uniform case, which is obtained using $S(z)=z)$. The running time of $M^{\prime}$ is still less than $T^{\prime}(m)=m^{k / \epsilon}$, and therefore the rest of the argument proceeds without change.

\subsection{Proofs of Theorems 1.1 and 1.8}

The proof of Theorem 1.1 in [11] follows a well-known strategy for constructing PRGs, which dates back to [3,14]. We now show how to simplify the analysis of any such construction, while pointing out that our simpler analysis also applies to a potentially-larger class of constructions.

In the well-known approach introduced by $[3,14]$, to construct a PRG they first construct a pseudoentropy generator (PEG), which takes as input a small random seed and outputs a string that appears to any efficient distinguisher as though it came from a distribution with high min-entropy; and then apply a seeded extractor to this pseudoentropic string. An extractor converts distributions with high statistical min-entropy to distributions that are statistically close to uniform, and the main idea is that we expect that a complexity-theoretic analogue of this statement will also hold: When the input distribution to the extractor looks to any efficient distinguisher as though it has high min-entropy, we intuitively expect its output distribution to look to any efficient distinguisher as close to uniform. This yields a PRG of the form

$$
G\left(s_{0}, s_{1}\right)=\operatorname{Ext}\left(G_{0}\left(s_{0}\right), s_{1}\right)
$$

\footnotetext{
${ }^{12}$ To be more accurate, $T$ solves a promise-problem wherein the "yes" instances are $x$ such that the gap between the acceptance probability of $M(x, \cdot)$ with random coins and the acceptance probability of $M(x, \cdot)$ with pseudorandom coins is less than $1 / 6$ and "no" instances are those in which the said gap is at least $1 / 8$. This problem can be solved probabilistically in time $O\left(n^{k+2 \epsilon}\right)$ (by sampling from the seeds of $G$ and from uniform coins for $M$ and comparing the two estimates), and the probabilistic algorithm can then be converted to a deterministic circuit of size poly $\left(n^{k}\right)$. See the proof of [8, Theorem 4.13] for details.
}

where $G_{0}$ is a pseudoentropy generator and Ext is an extractor.

The main challenge in materializing this approach is that constructing PEGs for standard notions of pseudoentropy (e.g., HILL pseudoentropy [14]) is challenging, and few constructions are known (see, e.g., [3, 14, 24]). Doron et al. [11] bypassed this obstacle by constructing a PEG for a weaker notion of pseudoentropy, called metric pseudoentropy ${ }^{13}$ however, they then faced the problem of proving that extracting from a string with high metric pseudoentropy yields a pseudorandom string, which required a lot of technical work. (In fact, to prove this they needed the PEG to "fool" a stronger and non-standard class of distinguishers; see [11].)

We show that any construction as in Eq. (2.1) is a special case of a class of constructions that can be analyzed in a different and significantly simpler way. To do so we follow an idea of Sipser [23], which was recently highlighted again in [13] and in a sequence of follow-up works concerning quantified derandomization (see, e.g., $[6,7,16,26,27])$. Specifically, consider any potential distinguisher $D:\{0,1\}^{N} \rightarrow\{0,1\}$ of size $O(N)$. We show that $G$ from Eq. (2.1) is $\epsilon$-pseudorandom for $D$, as follows:

(1) Non-standard reduction to quantified derandomization: Let $\bar{D}$ be a circuit that accepts its input $z$ if and only if $\operatorname{Pr}_{r}\left[\operatorname{Ext}(z, r) \in D^{-1}(1)\right] \in \mu \pm \epsilon / 2$, where $\mu=\operatorname{Pr}_{r}[D(r)=1]$. Note that $\bar{D}$ is not the circuit that is obtained by applying standard (extractor-based) error-reduction to $D$, but rather a circuit that tests whether or not its input causes the extractor to sample the event $D^{-1}(1)$ correctly, up to error $\epsilon / 2$. (In particular, the circuit $\bar{D}$ has the value $\mu$ hard-wired, but this does not cause a problem since $\bar{D}$ is only part of the analysis.) The two crucial points are that $\bar{D}$ accepts all but a tiny number of exceptional inputs (since Ext samples any event correctly, with extremely high probability); and that any $(\epsilon / 2)$ PRG $G_{0}$ for $\bar{D}$ yields an $\epsilon$-PRG $G(s, r)=\operatorname{Ext}\left(G_{0}(s), r\right)$ for D. ${ }^{14}$

(2) Solving the quantified derandomization problem: Thus, the only missing part in the proof is to construct a generator $G_{0}$ that is $(\epsilon / 2)$-pseudorandom for the extremely-biased circuit $\bar{D}$ (i.e., such that $\operatorname{Pr}_{s}[\bar{D}(G(s))=1] \geq 1-\epsilon / 2$ ). This is indeed a quantified derandomization problem, where the number of exceptional inputs (of $\bar{D}$ ) is dictated by the parameters of the extractor Ext.

Now, it follows from [3] that metric PEGs are equivalent to PRGs that solve the quantified derandomization problem (i.e., a metric PEG for min-entropy $k$ is equivalent to a PRG for distinguishers with at most $2^{k}$ exceptional inputs, where in both cases this parameter corresponds to the min-entropy of Ext; see [8, Proposition 3.11]). Thus, any PEG $G_{0}$ is also a PRG for quantified derandomization with the parameters induced by Ext, and therefore $G$ is indeed an $\epsilon$-PRG.

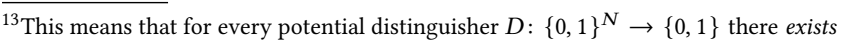
some distribution w over $\{0,1\}^{N}$ with high entropy such that $D$ does not distinguish between $\mathbf{w}$ and the output distribution of $G_{0}^{f}$ (for a precise definition see [11, Sec. 2]). ${ }^{14}$ To see this, call a string $z$ good if $\operatorname{Pr}_{r}\left[\operatorname{Samp}(z, r) \in D^{-1}(1)\right] \in \mu \pm \epsilon / 2$. Then, for any $\sigma \in\{0,1\}$ it holds that $\operatorname{Pr}_{s, r}\left[\operatorname{Samp}(G(s), r) \in D^{-1}(\sigma)\right] \leq$ $\operatorname{Pr}[G(s)$ is not good $]+\mu+\epsilon / 2<\epsilon$.
} 
The argument above shows that the composition of a metric PEG with an extractor as in Eq. (2.1) yields a PRG. ${ }^{15}$ We note that this argument applies to a potentially-larger class of constructions, compared to the class of constructions that can be analyzed via the pseudoentropy-based approach, since the known pseudoentropybased analysis requires the metric PEG to "fool" a stronger distinguisher class (see [11, Sec. 6]).

Applying the proof strategy in our setting. The alternative proof of Theorem 1.1 and our proof of Theorem 1.8 follow by applying the strategy above with a very simple construction of a PRG for quantified derandomization, which is presented in [8, Section 5.1]. This simple construction is inspired by a technical idea from Sipser's [23] original paper that introduced the approach of error-reduction and quantified derandomization, ${ }^{16}$ but to obtain the PRG that we need we instantiate the idea using more recent technical tools, such as locally list-decodable error-correcting codes (see [8, Section 5.1] for details).

As pointed out by Dean Doron, the latter construction is identical to a simplified construction of a pseudoentropy generator for the "higher-error" setting in a recent revision of [11]; and as pointed out by an anonymous reviewer, constructions of HSGs using essentially the same ideas date back to $[1,18]$.

The main difference between the proofs of Theorem 1.1 and Theorem 1.8 boils down to the construction of a circuit for the function $\bar{D}$. In more detail, note that $\bar{D}$ is a more complicated function than $D$, and that we need to solve the quantified derandomization problem for $\bar{D}$ rather than for $D$; intuitively, the overhead in implementing $\bar{D}$ as a circuit yields an overhead in our derandomization time.

The straightforward way of implmenting $\bar{D}$ yields derandomization either in cubic time or in quartic time, depending on the specific hardness hypothesis (see [8, Theorem 5.5]). An alternative way is to implement $\bar{D}$ using randomness, which mitigates the overhead and allows to obtain derandomization in quadratic time, at the cost of having to assume that the underlying hard function is hard for randomized SVN circuits (this yields the alternative proof of Theorem 1.1; see [8, Theorem 5.6]). For further details see [8, Section 5].

New light on bypassing the hybrid argument. As mentioned in Section 1.4, proofs of results such as Theorems 1.1 and 1.2 need to avoid a certain hybrid argument (see, e.g., [3, 12]). In [3, Sec 1.2] it was suggested that the proof strategy of "extracting from a pseudoentropic string" allows to bypass this barrier, since the analysis of a reconstruction procedure for a PEG might be easier than the analysis of a reconstruction procedure for a PRG. (To be more accurate, [3] suggested that the connection of pseudoentropy to unpredictability might be closer than the connection of pseudorandomness to unpredictability.)

Our main point is that in light of the above, the explanation can be reframed as suggesting that the analysis of PRGs for quantified derandomization might allow avoiding a hybrid argument more

\footnotetext{
${ }^{15}$ Since this holds for constructions that use a metric PEG, it also holds for constructions that use stronger PEGs.

${ }^{16}$ The original paper did not use the term quantified derandomization, which was only coined later by Goldreich and Wigderson [13].
}

easily than the analysis of PRGs for standard derandomization. ${ }^{17}$ This suggestion is at least as plausible as their original suggestion, since any proof that avoids a hybrid argument using the PEG-based approach also yields a proof that avoids a hybrid argument using the quantified-derandomization-based approach. Moreover, all the reconstruction procedures whose analyses avoid a hybrid argument in $[3,11]$ and in our work can be viewed as solving a corresponding quantified derandomization problem. ${ }^{18}$

We also point out the fact that in all three works, the reconstruction procedures that avoid a hybrid argument used "strong" resources (i.e., either used non-determinism or referred only to space-bounded computation, disregarding time). Thus, it is useful to recall that an additional potential explanation for the success so far might simply be that the analysis of such "strong" reconstruction procedures is easier.

\section{ACKNOWLEDGEMENTS}

We are very grateful to Oded Goldreich and to Ryan Williams for helpful feedback as well as detailed remarks on an early draft of the paper. We also thank Dean Doron, Igor Oliveira, Guy Rothblum, Amnon Ta-Shma, Avishay Tal, and Avi Wigderson for helpful conversations and feedback. The first author wants to thank Guy Rothblum for inviting him to the Weizmann Institute of Science, where part of the work was done. We also thank a helpful anonymous reviewer, who in particular pointed out the relevance of the works $[1,2,18]$.

This project has received funding from the European Research Council (ERC) under the European Union's Horizon 2020 research and innovation programme (grant agreement No. 819702). Lijie Chen is supported by NSF CCF-1741615, a Google Faculty Research Award, and an IBM Fellowship.

\section{REFERENCES}

[1] Alexander E. Andreev, Andrea E. F. Clementi, and José D. P. Rolim. 1998. A new general derandomization method. fournal of the ACM 45, 1 (1998), 179-213.

[2] Sergei Artemenko, Russell Impagliazzo, Valentine Kabanets, and Ronen Shaltiel. 2016. Pseudorandomness when the odds are against you. In Proc. 31st Annual IEEE Conference on Computational Complexity (CCC). Art. No. 9, 35.

[3] Boaz Barak, Ronen Shaltiel, and Avi Wigderson. 2003. Computational analogues of entropy. In Proc. 7th International Workshop on Randomization and Approximation Techniques in Computer Science (RANDOM). 200-215.

[4] Andreas Björklund. 2014. Determinant Sums for Undirected Hamiltonicity. SIAM 7. Comput. 43, 1 (2014), 280-299. https://doi.org/10.1137/110839229

[5] Manuel Blum and Silvio Micali. 1984. How to Generate Cryptographically Strong Sequences of Pseudo-random Bits. SIAM fournal of Computing 13, 4 (1984), 850-864.

[6] Lijie Chen, Ce Jin, and Richard Ryan Williams. 2020. Sharp threshold results for computational complexity. In Proc. 52nd Annual ACM Symposium on Theory of Computing (STOC).

[7] Lijie Chen and Roei Tell. 2019. Bootstrapping results for threshold circuits "just beyond" known lower bounds. In Proc. 51st Annual ACM Symposium on Theory of Computing (STOC). 34-41.

\footnotetext{
${ }^{17}$ This is consistent with the fact that the unconditionally-known constructions of PRGs for quantified derandomization indeed avoid a hybrid argument; see, e.g., [13, 26]. ${ }^{18}$ In the current work this fact is explicit, and in [11] this is because the reconstruction procedure is part of a construction of a metric PEG, which is equivalent to a PRG for quantified derandomization. In [3] there is no direct construction of a PEG or of a PRG for quantified derandomization, but the reconstruction procedures are ones that correspond to such construction: This is since the reconstruction algorithms in [3] transform a very biased distinguisher (of a pseudoentropic distribution $\mathbf{w}$ over $\{0,1\}^{n}$ from the uniform distribution $\mathbf{u}_{n}$ ) into an algorithm that predicts a bit in the pseudoentropic distribution with high success probability. (Indeed, the point is that the reconstruction algorithm only works for very biased distinguishers; see [3, Section 7] for further details.)
} 
[8] Lijie Chen and Roei Tell. 2020. Simple and fast derandomization from very hard functions: Eliminating randomness at almost no cost. Electronic Colloquium on Computational Complexity: ECCC 27 (2020), 148.

[9] Ruiwen Chen, Rahul Santhanam, and Srikanth Srinivasan. 2016. Average-case lower bounds and satisfiability algorithms for small threshold circuits. In Proc. 31st Annual IEEE Conference on Computational Complexity (CCC). 1:1-1:35.

[10] Marek Cygan, Stefan Kratsch, and Jesper Nederlof. 2018. Fast Hamiltonicity Checking Via Bases of Perfect Matchings. F. ACM 65, 3 (2018), 12:1-12:46. https: //doi.org/10.1145/3148227

[11] Dean Doron, Dana Moshkovitz, Justin Oh, and David Zuckerman. 2020. Nearly Optimal Pseudorandomness From Hardness. In Proc. 61st Annual IEEE Symposium on Foundations of Computer Science (FOCS).

[12] Bill Fefferman, Ronen Shaltiel, Christopher Umans, and Emanuele Viola. 2013. On beating the hybrid argument. Theory of Computing 9 (2013), 809-843.

[13] Oded Goldreich and Avi Widgerson. 2014. On derandomizing algorithms that err extremely rarely. In Proc. 46th Annual ACM Symposium on Theory of Computing (STOC). 109-118. Full version available online at Electronic Colloquium on Computational Complexity: ECCC, 20:152 (Rev. 2), 2013.

[14] Johan Håstad, Russell Impagliazzo, Leonid A. Levin, and Michael Luby. 1999. A Pseudorandom Generator from any One-way Function. SIAM fournal of Computing 28, 4 (1999), 1364-1396.

[15] Russell Impagliazzo and Avi Wigderson. 1999. P = BPP if E requires exponential circuits: derandomizing the XOR lemma. In Proc. 29th Annual ACM Symposium on Theory of Computing (STOC). 220-229.

[16] Valentine Kabanets and Zhenjian Lu. 2018. Satisfiability and derandomization for small polynomial threshold circuits. In Proc. 22th International Workshop on Randomization and Approximation Techniques in Computer Science (RANDOM). LIPIcs. Leibniz Int. Proc. Inform., Vol. 116. Art. No. 46, 19.

[17] Daniel Lokshtanov, Ramamohan Paturi, Suguru Tamaki, Ryan Williams, and Huacheng Yu. 2017. Beating brute force for systems of polynomial equations over finite fields. In Proc. 28th Annual ACM-SIAM Symposium on Discrete Algorithms (SODA). 2190-2202.
[18] Peter Bro Miltersen and N. V. Vinodchandran. 2005. Derandomizing ArthurMerlin games using hitting sets. Computational Complexity 14, 3 (2005), 256-279.

[19] Noam Nisan and Avi Wigderson. 1994. Hardness vs. randomness. Journal of Computer and System Sciences 49, 2 (1994), 149-167.

[20] Ramamohan Paturi, Pavel Pudlák, Michael E. Saks, and Francis Zane. 2005. An improved exponential-time algorithm for k-SAT. F. ACM 52, 3 (2005), 337-364. https://doi.org/10.1145/1066100.1066101

[21] Ran Raz, Omer Reingold, and Salil Vadhan. 2002. Extracting all the randomness and reducing the error in Trevisan's extractors. Journal of Computer and System Sciences 65, 1 (2002), 97-128.

[22] Ronen Shaltiel and Christopher Umans. 2005. Simple extractors for all minentropies and a new pseudorandom generator. fournal of the ACM 52, 2 (2005), $172-216$.

[23] Michael Sipser. 1988. Expanders, randomness, or time versus space. Vol. 36. 379-383.

[24] Madhu Sudan, Luca Trevisan, and Salil Vadhan. 2001. Pseudorandom generators without the XOR lemma. Fournal of Computer and System Sciences 62, 2 (2001), 236-266.

[25] Amnon Ta-Shma, David Zuckerman, and Shmuel Safra. 2006. Extractors from Reed-Muller codes. fournal of Computer and System Sciences 72, 5 (2006), 786-812.

[26] Roei Tell. 2017. Improved Bounds for Quantified Derandomization of ConstantDepth Circuits and Polynomials. In Proc. 32nd Annual IEEE Conference on Computational Complexity (CCC). 18:1 - 18:49.

[27] Roei Tell. 2018. Quantified Derandomization of Linear Threshold Circuits. In Proc. 50th Annual ACM Symposium on Theory of Computing (STOC). 855-865.

[28] Christopher Umans. 2003. Pseudo-random generators for all hardnesses. Fournal of Computer and System Sciences 67, 2 (2003), 419-440.

[29] Richard Ryan Williams. 2016. Strong ETH breaks with Merlin and Arthur: short non-interactive proofs of batch evaluation. In Proc. 31st Annual IEEE Conference on Computational Complexity (CCC). Vol. 50. Art. No. 2, 17.

[30] Andrew C. Yao. 1982. Theory and Application of Trapdoor Functions. In Proc. 23rd Annual IEEE Symposium on Foundations of Computer Science (FOCS). 80-91. 